\title{
Computation method of geometry die of stretch forming press
}

\author{
Konstantin Bormotin ${ }^{1, *}$, and Win Aung ${ }^{1}$ \\ ${ }^{1}$ Komsomolsk-na-Amure State University, 681013, Komsomolsk-on-Amur, Russia
}

\begin{abstract}
Mathematical models and numerical methods for solving inverse problems of shell forming by means of stretching on a die have been developed. The algorithms implemented in MSC.Marc allow to calculate the required punch shape. The results of simulation of the stretching technology are presented.
\end{abstract}

\section{Introduction}

At present, the aircraft industry enterprises are equipped with stretch-wrap forming equipment with numerical program control, which has wide technological capabilities. The control program of the stretching-wrap press carries out a "wrapping" of the sheet workpiece of the stretching die under differentiated tension due to the movement of the clamping devices. The stretching process is a forming operation of sheet punching. The main thing in the forming process is a provide obtain a geometric shape of the shell.

When wrapping with stretching, the defomation of the sheet preform at each moment of time is localized in a narrow region along the boundary of contact with the surface of the punch. This leads to local thinning of the sheet, loss of stability (folding). Methods are developed for stretching shells of complex shapes, with a smaller probability of localization of deformation due to kinematic displacements of the working organs of the stretching press. In particular, we propose a method for manufacturing double curvature shell on the $\mathrm{CNC}$ stretching equipment, which ensures equalization of the strain field, a reduction in the number of transitions, and a reduction in the laboriousness of the forming process [1]. To increase the stability of the forming process of the shells of a convex-concave shape, a technological technique based on the use of a billet with an increased width is proposed [2]. To estimate the parameters of the stretch-wrap forming, various modeling methods are considered [3].

This equipment has wide technological capabilities, for example it can be used for forming, both shells and profile blanks [4]. Automated forming of parts on the press of the transverse stretching FET requires the development of a control program and an electronic model of the stretching punch. The S3F-FET software supplied with this equipment provides information only on the parameters of the working elements of the press. To simulate the stretching processes in the CAE-system, the boundary conditions of the trajectory of motion are calculated using the developed program [5].

\footnotetext{
* Corresponding author: cvmi@knastu.ru
} 
The development of technical solutions in the methods of forming does not guarantee obtaining high accuracy of the final geometric part shape. The precision of the shape of the part obtained by the processing of materials by pressure at specified process parameters depends on the accuracy of the computed and manufactured form of the tool (punch), which determines the anticipatory shape of the panel. The lead form should provide the given residual curvature of the panel after its release from the force tool. In this connection, the opposite problem arises: to determine the shape of the tooling, creating such anticipatory curvature, which ensures the given residual shape of the panel after unloading.

\section{Formulation and method for solving problem}

Let $V \subset R^{3}$ be a bounded domain with a sufficiently regular boundary $S$. The contact surface of die with deformable body is designated through $S_{c}$. The surface of the workpiece in contact with the clamping devices is indicated by $S_{b}$. Denote by $u=\left(u_{1}, u_{2}, u_{3}\right), \quad \tilde{u}=\left(\tilde{u}_{1}, \tilde{u}_{2}, \tilde{u}_{3}\right), \quad \bar{u}=\left(\bar{u}_{1}, \bar{u}_{2}, \bar{u}_{3}\right) \quad$ - the vectors of current, residual displacements of deformable body and the vectors displacements of the die surface points; $u, \tilde{u} \in\left[W_{2}^{1}(Q)\right]^{3}, Q=V \times\{0 \leq t \leq T\}, \bar{u} \in\left[W_{2}^{1}\left(Q_{c}\right)\right]^{3}, Q_{c}=S_{c} \times\{0 \leq t \leq T\}$. Here $t$ is the parameter of deformation. The symbol $(\cdot,)_{S}$ denotes the inner product in $L_{2}(S)$ : $(u, v)_{S}=\int_{S} \sum_{i=1}^{3} u_{i} v_{i} d S$. The corresponding norm is given by $\|u\|_{S}=\sqrt{(u, u)_{S}}=\left(\int_{S} \sum_{i=1}^{3} u_{i}^{2} d S\right)^{1 / 2}$.

The inverse problem of a kinematic forming by contact rigid stamps can be formulated similarly to [6-8] in the form of a quasistatic variational principle with the functional:

$$
\begin{aligned}
& J(\dot{\bar{u}}, \dot{u}, \dot{\tilde{u}})=\frac{1}{2 \varepsilon_{1}}\left\|\dot{\bar{u}}-\dot{\bar{u}}^{*}\right\|_{S_{c}}{ }^{2}+W_{c}+\frac{1}{2 \varepsilon_{2}}\left\|\dot{u}-\dot{u}^{*}\right\|_{S_{b}}{ }^{2}+a(\dot{u}, \dot{u})+ \\
& +a(\dot{\tilde{u}}, \dot{\tilde{u}})+\frac{1}{2 \varepsilon_{3}}\left\|\dot{\tilde{u}}-\dot{\tilde{u}}^{*}\right\|_{S}{ }^{2}, \varepsilon_{1}>0, \varepsilon_{1} \rightarrow 0, \varepsilon_{2}>0, \varepsilon_{2} \rightarrow 0,
\end{aligned}
$$

where $\dot{\tilde{u}}^{*}, \dot{\bar{u}}^{*}, \dot{u}^{*}$ - is a given residual displacement rate and current contact displacement rate of die and shell; $W_{c}$ - the quasistatic contact potentials received by imposing of contact conditions on the equations of bodies motion by method of multipliers of Lagrange or by method of penal functions [9] and differentiation on $t$; potential form are given by

$$
\begin{aligned}
& a(\dot{u}, \dot{v})=\int_{V} \frac{\partial E\left(\dot{u}_{i, j}\right)}{\partial \dot{u}_{i, j}} \dot{v}_{i, j} d V, a(\dot{\tilde{u}}, \dot{\tilde{v}})=\int_{V}\left(\frac{\partial \tilde{E}\left(\dot{\tilde{u}}_{i, j}\right)}{\partial \dot{\tilde{u}}_{i, j}}\right) \dot{\tilde{v}}_{i, j} d V, \\
& E\left(\dot{u}_{i, j}\right)=\frac{1}{2} c_{i j p l} \dot{\varepsilon}_{i j} \dot{\varepsilon}_{k l}-c_{i j p l} \dot{\varepsilon}_{i j} \dot{\varepsilon}_{p l}^{p}+\frac{1}{2} \sigma_{i j} \dot{u}_{p, i} \dot{u}_{p, j}, \quad \tilde{E}\left(\dot{\tilde{u}}_{i, j}\right)=\frac{1}{2} c_{i j p l} \dot{\tilde{\varepsilon}}_{i j} \dot{\tilde{\varepsilon}}_{p l}-c_{i j p l} \dot{\tilde{\varepsilon}}_{i j} \dot{\varepsilon}_{p l}^{p}+\frac{1}{2} \rho_{i j} \dot{\tilde{u}}_{p, i} \dot{\tilde{u}}_{p, j},
\end{aligned}
$$

$c_{i j k l}$ - are the components of the elastic constant tensor; $\dot{\varepsilon}_{k l}^{p}$ - are the plastic strain rates;

$\dot{\varepsilon}_{i j}=\frac{1}{2}\left(\dot{u}_{i, j}+\dot{u}_{j, i}+\dot{u}_{p, i} u_{p, j}+u_{p, i} \dot{u}_{p, j}\right)$,

$$
\dot{\tilde{\varepsilon}}_{i j}=\frac{1}{2}\left(\dot{\tilde{u}}_{i, j}+\dot{\tilde{u}}_{j, i}+\dot{\tilde{u}}_{p, i} \tilde{u}_{p, j}+\tilde{u}_{p, i} \dot{\tilde{u}}_{p, j}\right) ;
$$

$i, j, k, l=1,2,3$. 
By consideration of the inverse problem with functional in the form of (1) it is supposed that in the considered time $t$ there is a contact of rigid stamp with deformable body, otherwise these contact potentials shouldn't enter functional.

Taking into account discretization of the variation (1) the finite element equations for the solution of a contact problem are formed $[9,10]$

$$
{ }^{t+\Delta t} \mathbf{K}^{(i-1)} \Delta \mathbf{U}^{(i)}={ }^{t+\Delta t} \mathbf{R}^{(i-1)},{ }^{t+\Delta t} \tilde{\mathbf{K}}^{(i-1)} \Delta \hat{\mathbf{U}}^{(i)}={ }^{t+\Delta t} \hat{\mathbf{R}}^{(i-1)},
$$

where ${ }^{t+\Delta t} \mathbf{K}^{(i-1)},{ }^{t+\Delta t} \tilde{\mathbf{K}}^{(i-1)}$ - are the tangent stiffness matrices (in matrices ${ }^{t+\Delta t} \mathbf{K}^{(i-1)}$ are already included the additional elements which are formed from contact restrictions), ${ }^{t+\Delta t} \mathbf{R}^{(i-1)},{ }^{t+\Delta t} \hat{\mathbf{R}}^{(i-1)}$ - the vector of internal and external forces. The top indexes $t+\Delta t$ of quantity indicate time for which it is calculated. The top indexes $(i-1)$ indicate number of iteration at correction of the solution by Newton-Rafson's method. The solution of the following step is found on a formula ${ }^{t+\Delta t} \mathbf{U}={ }^{t} \mathbf{U}+\Delta \mathbf{U}$.

As a result of the solution of the first problem of the given movements we receive the deformed model with distribution of stress and strain. The second problem on the basis of data on initial stress and strain determines unloading movements. After that it is possible to find residual nodal movements $\tilde{\mathbf{U}}=\mathbf{U}+\hat{\mathbf{U}}$.

Several procedures have been developed to treat contact problems including the use of Perturbed or Augmented Lagrangian methods, penalty methods, [9, 11] and direct constraints. In the latter case in MSC.Marc, when the contact occurs, the degrees of freedom are transformed to a local system and a constraint is imposed such that $\Delta u_{\text {normal }}=\mathbf{v} \cdot n$, where $\mathbf{v}$ is the prescribed velocity of the rigid surface.

The iterative method for solving the inverse forming problem in displacement of a contact surface of die is offered

$$
\begin{aligned}
& \bar{u}_{i}^{k+1}=\bar{u}_{i}^{k}+\alpha^{k}\left(\tilde{u}_{i}^{*}-\tilde{u}_{i}^{k}\right)+\beta^{k}\left(\bar{u}_{i}^{k}-\tilde{u}_{i}^{k}\right) \text { on } S_{c}, \\
& u_{i}^{k+1}=u_{i}^{k}+\alpha^{k}\left(\tilde{u}_{i}^{*}-\tilde{u}_{i}^{k}\right)+\beta^{k}\left(u_{i}^{k}-\tilde{u}_{i}^{k}\right) \text { on } S_{b},
\end{aligned}
$$

where $0<\alpha^{k}<2, \beta^{k} \rightarrow 0$ at $k \rightarrow \infty, i=1,2,3$.

\section{Numerical results of the problem solution}

The problem of calculating the shape of the punch, creating such a lead curvature of the panel during the stretching, which provides the given residual shape after unloading, is considered. Simulation of the formation of a double-curvature panel with a thickness of 2 $\mathrm{mm}$ is carried out by CAE in MSC.Marc (fig.1). The dimensions of the workpiece are $1750 \times 750 \times 2 \mathrm{~mm}$. The workpiece has the properties of material 1163T. The material is isotropic and its elastic characteristics are the same under tension and compression and are equal to the following values: Young's modulus $E=7454 \frac{\mathrm{kg}}{\mathrm{mm}^{2}}$, Poisson's ratio v $=0.34$, yield strength $\sigma_{T}=29.85 \frac{\mathrm{kg}}{\mathrm{mm}^{2}}$, linear hardening modulus $E_{T}=200.75 \frac{\mathrm{kg}}{\mathrm{mm}^{2}}$. The deformation process takes place in two stages: wrapping the punch and stretching the panel by $1 \%$. After unloading, the calculated residual displacement field of the panel is used in (2). The change in the punch geometry is carried out by solving the problem of surface 
deformation by the displacement field (2). These tasks are solved at each iteration, until the necessary accuracy is ensured.

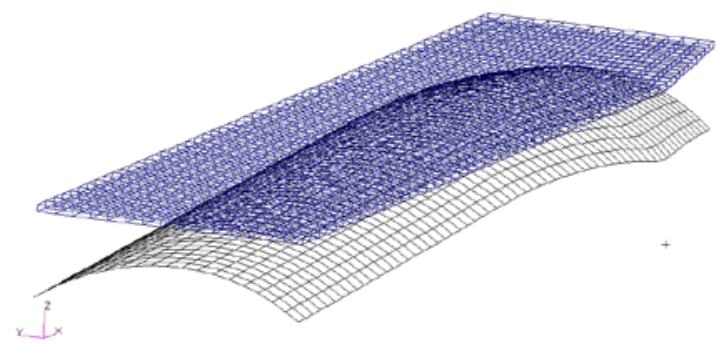

Fig. 1. Finite element model of the workpiece and a surface with a given curvature

In order to simplify the problem, the contact conditions simulate slippage without friction. As the initial displacement of the surface of the punch (2), the distance to the surface with the required curvature is taken $\bar{u}_{i}^{0}$, and $u_{i}{ }^{0}$ - the edges of the workpiece are moved to the current surface of the punch. Residual displacements in the iteration method are determined at the nodes of the deformed body nearest to the projections of the punch surface points. The iterative method is implemented in the MSC.Patran, MSC.Marc system using a number of user procedures [11].

As a result of calculation, the geometry of the punch is found, which reduces the deviations of the workpiece from the given shape after the stretching and unloading (Fig. 2, 3). Fig. 2 shows the residual shape of the preform after the stretching along the initial surface of the punch, in Fig.3 - the residual shape of the workpiece after stretching the surface of the punch, obtained by the iteration method for 3 iterations. Thus, deviations from the given geometry decreased by 1.5 times.

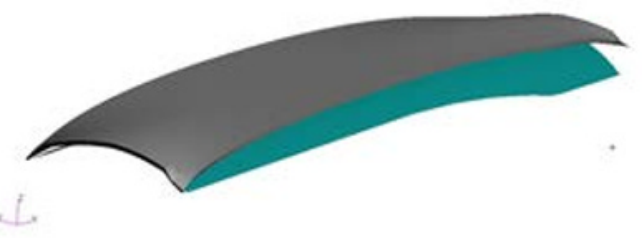

Fig. 2. Residual configuration of the workpiece after the first iteration in comparison with the given surface

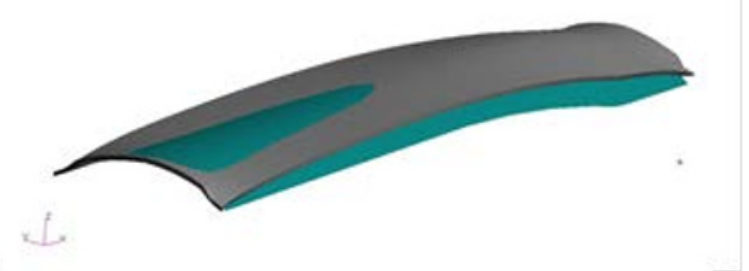

Fig. 3. Residual configuration of the workpiece after the third iteration in comparison with the given surface 


\section{Conclusions}

Program-controlled stretch-wrap presses provide the implementation of various shell shaping schemes. The choice of rational schemes is carried out depending on the geometric parameters of the shell, the limiting coefficient of the stretch and the type of the stretchwrap equipment. developed algorithm is proposed for to refine the geometry of the punch during shaping with the known stretching technology. In this problem, the simplest loading law of the shell is taken and it was not optimized.

This research was financially supported by the Russian Foundation for Basic Research (16-31-60038 mol_a_dk), Council for grants of the President of the Russian Federation (MD-4117.2018.1).

\section{References}

1. M. Molod, Bulletin of Voronezh State Technical University, № 12-2, 7, 62-64,(2011)

2. V. Mikheev, Izvestiya of the Samara Scientific Center of the Russian Academy of Sciences, №2, 6, 408-413, (2004)

3. R. Krupsky, A. Krivenok, A. Stankevitch, S. Feoktistov, S. Belykh, Scientific notes of KnAGTU, № II-1(14), 4-8, (2013)

4. V. Mironenko, A. Cheslavskaya, S. Belykh, Scientific notes of KnAGTU, № II-1 (18), 13-18, (2014)

5. R. Krupskiy, A. Krivenok, A. Stankevich, S. Belykh, V. Mironenko, // Bulletin IrSTU №9 (92), 40-44, (2014)

6. K. Bormotin, Computational mathematics and mathematical physics, №12, 53, 19081915 (2013)

7. K. Bormotin, Computational methods and programming, 15, 222-228 (2014)

8. K. Bormotin, S. Belykh and Win Aung, Computational methods and programming, 17, 258-267 (2016)

9. P. Wriggers, Computational Contact Mechanics ( 2006)

10. K.-J. Bathe, Finite element procedures (1982)

11. Marc 2016, Vol A: Theory and User Information, MSC.Software Corporation http://www.mscsoftware.com/product/marc. 